\title{
Adenomatoid Odontogenic Tumor: An Extra Follicular Variant in the Mandible of 12 Years Old Pediatric Female Patient
}

\section{Manjul Tiwari*}

School of Dental Sciences, Sharda University, Uttar Pradesh, India

\begin{abstract}
Adenomatoid odontogenic tumor [AOT] is a rare odontogenic tumor which is often misdiagnosed as odontogenic cyst. AOT accounts for about $1 \%$ until $9 \%$ of all odontogenic tumors. It is predominantly found in young female patients, located more often in maxilla and most often associated with an unerupted permanent tooth. However, AOT frequently resemble other odontogenic lesions such as dentigerous cysts or ameloblastoma. For illustration a rare case of an AOT in the mandible in a 12-year-old female patient is presented.
\end{abstract}

Keywords: Adenomatoid odontogenic tumor; Extra-follicular; Mandible

\section{Introduction}

Odontogenic tumors are lesions that derive from the toothproducing tissues or their remnants that remain entrapped either within the jawbones or into the adjacent soft tissues. From a biological point of view, some of these lesions represent hamartomas with varying degrees of differentiation, while the rest are benign or malignant neoplasms with variable aggressiveness and potential to develop metastasis.

At present, it is known that the potential sources to develop an odontogenic tumor are varied, and these include:

1. The pre-functional dental lamina [odontogenic epithelium with ability to produce a tooth], which is more abundant for obvious reasons distally to the lower third molars.

2. The post functional dental lamina, a concept that covers those epithelial remnants such as Serre's epithelial rests, located within the fibrous gingival tissue; the epithelial cell rests of Malassez in the periodontal ligament and the reduced enamel organ epithelium, which covers the enamel surface until tooth eruption.

3. The basal cell layer of the gingival epithelium, which originally gave rise to the dental lamina.

4. The dental papilla, origin of the dental pulp, which has the potential to be induced to produce odontoblasts and synthesize dentin and/or dentenoid material.

5. The dental follicle.

6. The periodontal ligament, which has the potential to induce the production of fibrous and cemento osseous mineralized material [1].

The AOT appears in three clinico-topographic variants: 1 . follicular 2. Extra-follicular 3. Peripheral all of which have identical presentation. The follicular variant is associated with the crown of an embedded tooth most frequently a permanent canine. The extra follicular variant has neither a pericoronal nor any other relationship to an embedded tooth. The peripheral type is located in the gingival mucosa and appears clinically as a gingival hyperplasia or fibrous epulis [2].

Histological examination reveals a thick capsule of fibrous connective tissue. The tumor fills the central cavity, there is little stroma. Tumor cells frequently form ball-like structures referred to as "rosettes" [3].
In this article, I am presenting a clinico-pathological observation of the extra-follicular variety of this infrequent benign tumor, affecting the anterior area of the mandible, in a 12-year-old patient.

\section{Case Report}

A 12 years-old female patient visited with chief complaint of an asymptomatic swelling in Mandibular anterior teeth region since last 1.5-2 months which was gradually increased up to the present size. The history revealed that the swelling had started insidiously with no evidence of any trauma. Medical, Surgical, personal and Family histories were not noteworthy. General physical examination revealed no abnormalities. Clinical examination revealed bilateral facial asymmetry involving the symphysis region causing protuberance of chin. The swelling was appearing to be $5 \times 4 \mathrm{cms}$ in diameter and firm too hard in consistency. Overlying skin was tensed and swelling was extended anterio-posteriorly from the region of 31-44 and superiorinferiorly from the vermilion border of lower lip up to the lower border of mandible.

Intra- oral examination revealed a firm to hard swelling measuring $5 \times 5 \mathrm{cms}$ with diffused borders involving the Mandibular labial vestibule in the region of 31-44 region causing vestibular obliteration (Figure 1).

Panoramic radiograph revealed a unilocular, diffuse radiolucency containing many small, irregular bony trabeculae, traversing the radiolucent area with displacement of Mandibular anterior teeth (Figure 2). Differential diagnosis was calcifying epithelial odontogenic tumor and adenomatoid odontogenic tumor (Figures 3 and 4).

\section{Discussion}

Adenomatoid odontogenic tumor $[\mathrm{AOT}]$ is a relatively uncommon distinct odontogenic neoplasm comprises only 0.1 per cent of tumors and cysts of the jaw and 3 per cent of all odontogenic tumours [4],

*Corresponding author: Manjul Tiwari, Assistant professor, Department of Oral Pathology and Microbiology, School of Dental Sciences, Sharda University, Uttar Pradesh, India; E-mail: manjultiw@gmail.com

Received: January 04, 2016; Accepted: January 14, 2016; Published: January 24 2016

Citation: Tiwari M (2016) Adenomatoid Odontogenic Tumor: An Extra Follicular Variant in the Mandible of 12 Years Old Pediatric Female Patient. Oral health case Rep 1:104. doi:10.4172/2471-8726.1000104

Copyright: (c) 2016 Tiwari M. This is an open-access article distributed under the terms of the Creative Commons Attribution License, which permits unrestricted use, distribution, and reproduction in any medium, provided the original author and source are credited. 
Citation: Tiwari M (2016) Adenomatoid Odontogenic Tumor: An Extra Follicular Variant in the Mandible of 12 Years Old Pediatric Female Patient. Oral health case Rep 2:104. doi:10.4172/2471-8726.1000104

Page 2 of 2

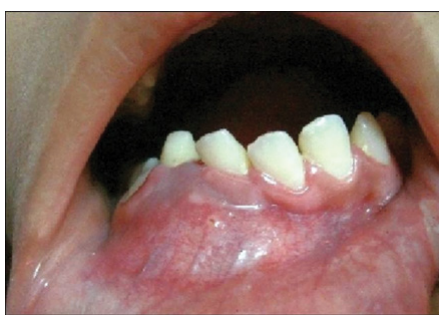

Figure 1: Intra oral examination.

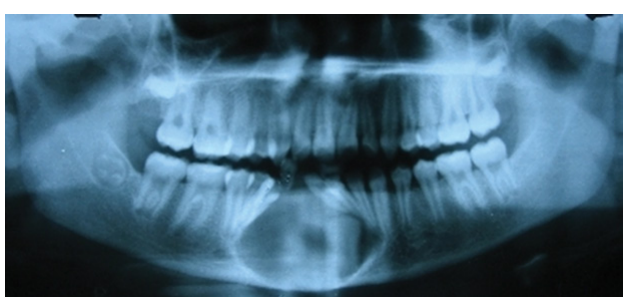

Figure 2: Panoramic radiograph.

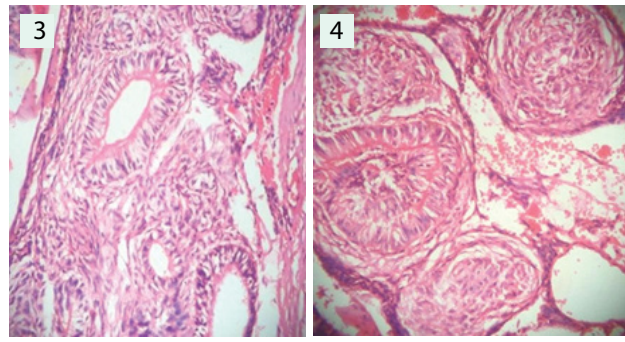

Figures 3 and 4: Calcifying epithelial odontogenic tumor and adenomatoid odontogenic tumor.

first described by Steensland in 1905 [1]. However, a variety of terms have been used to describe this tumor. Unal et al., [2] produced a list containing all nomenclatures for AOT reported in the literatures. Many different names like adeno-ameloblastoma, ameloblastic adenomatoid tumor, adamantinoma, epithelioma adamantinum or teratomatous odontoma have been used before to define the lesion currently called AOT $[4,5]$.

The maxilla is involved nearly twice as frequently as the mandible. Subsequently in cases reported by various authors these tumors are described as intraoral-extraoral swellings in the maxilla. Jorge GK Handschel et al., [6,7] reported AOT in mandible which is not the common site of occurrence for this tumor. They are more frequently seen in females during the second decade of life. Apart from a few exceptional cases the tumor is associated with unerupted teeth. The unerupted teeth are usually canine or lateral incisors. Irregular root resorption and dilacerations within the lesion are only infrequently reported in the literature. The lesion usually appears radio graphically as a unilocular lesion but at leas four cases of multilocular appearance have been reported. Radiopacities are often seen in the pericoronal radiolucency. Histopathologically AOT is usually surrounded by a well developed connective tissue capsule and the tumor is composed of spindle shaped or polygonal cells forming sheets and whorled masses in a scanty connective tissue stroma. The characteristic duct like structures is lined by a single row of columnar epithelial cells, the nuclei of which are polarized away from the central lumen $[4,5]$.

Interestingly the presented case had some unusual clinical, radio graphical and histological features that distinguished it from most normal types of AOT. Firstly the tumor is usually present in maxilla and associated with impacted maxillary canine but in the present case it was present in anterior mandible with no impacted tooth. Secondly AOT are slow growing and smaller in size but in the present case it was rapidly increasing in its size and of approximately $4 \times 5 \mathrm{cms}$ in size. Thirdly radiographic examination usually reveals a well defined radiolucency surrounding the impacted tooth but in the present case a diffuse radiolucency containing many small, irregular bony trabeculae, traversing the radiolucent area with displacement of Mandibular anterior teeth was observed. Fourthly a fibrous capsule is present around the tumor but this feature was absent in the present case, suggesting its aggressive nature [8,9]. Thus on the bases on currently available evidence and the findings the case, was diagnosed as extrafollicular variety of AOT.

\section{References}

1. Steenland HS (1905) Epithelioma adamantinum. J Exper Med 6: 377-389.

2. Unal T, Cetingul E, Gunbay T (1995) Peripheral Adenomatoid odontogenic tumor: Birth of a term. J Clin Pediatr Dent 19: 139-142

3. Dayi E (1997) Adenomatoid odontogenic tumour (adenoameloblastoma). Case report and review of the literature. Australian Dental Journal 42: 315-8

4. Philipsen HP, Reichart PA, Nikai H (1998) The Adenomatoid Odontogenic Tumor: An Update. Oral Med Pathol 2: 55-60

5. Dunlap C (2001) odontogenic tumors: the short version.

6. Handschel JGK, Depprich RA, Zimmermann AC, Braunstein S, Kübler NR (2005) Adenomatoid odontogenic tumor of the mandible: review of the literature and report of a rare case Head \& Face Medicine 1: 3.

7. Jivan V, Altini M, Meer S, Mahomed F (2007) Adenomatoid Odontogenic Tumor (AOT) Originatingin a Unicystic Ameloblastoma: A Case Report Head and Neck Pathol 1: 146-149.

8. Mosqueda-Taylor A (2008) New findings and controversies in odontogenic tumors Med Oral Patol Oral Cir Bucal 13: e555-8.

9. Garg D, Palaskar S, Shetty VP, Bhushan A (2009) Adenomatoid Odontogenic Tumor-hamartoma or true neoplasm: a case report. Journal of oral science 51 : 155-159. 